CLINICAL STUDY

\title{
Long-term re-evaluation of primary aldosteronism after medical treatment reveals high proportion of normal mineralocorticoid secretion
}

\author{
Barbara Lucatello, Andrea Benso, Isabella Tabaro, Elena Capello, Mirko Parasiliti Caprino, Lisa Marafetti ${ }^{1}$, \\ Denis Rossato ${ }^{2}$, Salvatore E Oleandri ${ }^{3}$, Ezio Ghigo and Mauro Maccario \\ Division of Endocrinology, Diabetology and Metabolism, Department of Internal Medicine, University of Turin, Az. Ospedaliera Città della Salute e della \\ Scienza, sede Molinette, C.so Dogliotti 14, 10126 Torino, Italy, ${ }^{1}$ Diabetes and Metabolism Unit, ASL Torino 5, Chieri, Italy, ${ }^{2}$ Department of Radiology, \\ University of Turin, Torino, Italy and ${ }^{3}$ Division of Endocrinology and Metabolism, SS Annunziata Hospital, Savigliano, Italy \\ (Correspondence should be addressed to M Maccario; Email: mauro.maccario@unito.it)
}

\begin{abstract}
Objective: In most cases of primary aldosteronism (PA), an adrenal aldosterone-secreting tumor cannot be reasonably proven, so these patients undergo medical treatment. Controversial data exist about the evolution of PA after medical therapy: long-term treatment with mineralocorticoid antagonists has been reported to normalize aldosterone levels but other authors failed to find remission of mineralocorticoid hypersecretion. Thus, we planned to retest aldosterone secretion in patients with medically treated PA diagnosed at least 3 years before.

Design: Retrospective, cross-sectional study.

Methods: The same workup for PA as at diagnosis (basal aldosterone to renin activity ratio (ARR) and aldosterone suppression test) was performed after stopping interfering drugs and low-salt diet, in 34 subjects with PA diagnosed between 3 and 15 years earlier, by case finding from subgroups of hypertensive patients at high risk for PA. Criteria for persistence of PA were the same as at diagnosis $($ ARR $(\mathrm{pg} / \mathrm{ml}$ per ng per $\mathrm{ml}$ per $\mathrm{h})>400$, aldosterone $>150 \mathrm{pg} / \mathrm{ml}$ basally, and $>100 \mathrm{pg} / \mathrm{ml}$ after saline infusion) or less restrictive.

Results: PA was not confirmed in 26 (76\%) of the patients and also not in 20 (59\%) using the least restrictive criteria suggested by international guidelines. Unconfirmed PA was positively associated with female sex, higher potassium levels, longer duration of hypertension, and follow-up, but not with adrenal mass, aldosterone levels at diagnosis, and treatment with mineralocorticoid antagonists.

Conclusions: This study suggests that mineralocorticoid hyperfunction in patients with PA after medical treatment may decline spontaneously. Higher potassium concentration and duration of treatment seem to increase the probability of this event.
\end{abstract}

European Journal of Endocrinology 168 525-532

\section{Introduction}

Primary aldosteronism (PA) has been recognized as the most frequent cause of secondary hypertension in middle-aged adults (1). When caused by an aldosteronesecreting adrenal adenoma, $\mathrm{PA}$ is a pathological condition that can be easily cured by unilateral adrenalectomy. Nevertheless, as the lateralization of the mineralocorticoid hypersecretion cannot be demonstrated with certainty, patients with PA, even bearing an adrenal mass, do not undergo surgical treatment. These cases are treated with mineralocorticoid receptor antagonists unless there are contraindications or side effects. Since the widespread screening of hypertensive patients, regardless of potassium levels, the medically treated subtype has now become the most frequent PA subtype $(2,3)$, but scanty data are available in the literature about its clinical course. In the 1980s, Biglieri et al. (4) proposed an evolutionary hypothesis of PA, suggesting that the disease could have started as a low-renin essential hypertension, progressed to bilateral hyperplasia of the zona glomerulosa, unilateral hyperplasia, and finally to unilateral adenoma. However, no studies have provided evidence for this theory nowadays. Moreover, patients with unilateral adenoma are usually the youngest among patients with PA $(5,6)$, and cases of unilateral adenoma in newborns have also been described (7).

Recent studies suggest a possible remission of PA during prolonged medical treatment with antagonists of mineralocorticoid receptor $(8,9)$. However, recently, a reevaluation of aldosterone secretion in 37 patients with idiopathic hyperaldosteronism from the Munich Conn's Registry has reported only two cases of 'normalization' (10). Furthermore, to our knowledge, there 
are no data on the evolution of PA without specific anti-aldosterone treatment. For this reason, we planned to reevaluate the spontaneous and dynamic mineralocorticoid secretion, years after the diagnosis, in patients with medically treated PA, either treated or not with mineralocorticoid receptor antagonists.

\section{Materials and methods}

\section{Patients}

Before 2008, among the hypertensive patients diagnosed with PA at our Center for Clinical Management of Hypertension, 63 patients were judged not to have the indication for surgery. For all these subjects, PA was suspected according to international guidelines (11) (hypokalemia, hypertension with adrenal mass, resistant hypertension, juvenile hypertension, and early cardiovascular events in hypertensive patients). We studied 34 out of these 63 patients, at least 3 years after diagnosis (18 males and 16 females, mean follow-up 8.4 years, range 3-15 years). The remaining 29 patients were not tested because they did not give consent or were lost to follow-up. Recruited patients did not differ from not-recruited ones for sex, aldosterone, and potassium levels at diagnosis, or for the presence of an adrenal mass. Recruited patients were only slightly younger $(58 \pm 8.5$ vs $64 \pm 11$ years, mean \pm s.D.; $P=0.02$ ) and presented a shorter duration of hypertension at diagnosis $(8 \pm 7$ vs $14 \pm 12$ years; $P=0.03)$.

All patients studied were regularly followed at our center with 6- to 18-month outpatient visits (or more frequently if insufficient blood pressure control) to assess compliance to the behavioral and pharmacological treatment, pressure control on home measurements, hemodynamic compensation, renal function, and the management of cardiovascular risk factors.

\section{Measures at diagnosis}

Diagnosis of PA at referral had been made as the Endocrine Society suggests in the Clinical Practice Guideline (11); all the following conditions had to be met: i) plasma aldosterone concentration (PAC) over $150 \mathrm{pg} / \mathrm{ml}$, ii) plasma renin activity (PRA) under $1 \mathrm{ng} / \mathrm{ml}$ per $\mathrm{h}$, iii) aldosterone to renin ratio (ARR) $>400$, and iv) PAC over $100 \mathrm{pg} / \mathrm{ml}$ after administration of $2 \mathrm{l}$ of saline in $4 \mathrm{~h}$ ( $\mathrm{NaCl}$ test) or over $60 \mathrm{pg} / \mathrm{ml}$ after 4 days of fludrocortisone treatment with $0.4 \mathrm{mg} /$ day (fludrocortisone test). All patients had been diagnosed during a free diet without salt restriction during the week before the biochemical evaluation. All subjects were given a potassium supplementation if hypokalemic (i.e. potassium $<3.5 \mathrm{mmol} / \mathrm{l}$ ) and were studied after adequate (30-40 days) withdrawal of interfering drugs. Interfering drugs were considered diuretics, ACE inhibitors, angiotensin II receptor antagonists, $\beta$-blockers, clonidine, and aldosterone receptor antagonists. Doxazosin and/or a long-acting calcium channel blocker was prescribed to control hypertension and was continued up to the end of evaluation. Blood samples for PAC and PRA were obtained in sitting position between 0800 and $1000 \mathrm{~h}$.

Imaging of adrenals (CT scan or magnetic resonance imaging) was also obtained in all subjects: ten patients had a unilateral lesion, three patients had bilateral lesions, and in the remaining 21 the adrenal morphology was normal. All patients were advised to undergo an adrenal vein sampling (AVS) in search of a lateralized secretion of aldosterone, but 14 patients declined (three with a unilateral and one with bilateral lesions). AVS could not clearly demonstrate a lateralization of aldosterone secretion (lateralization index $<4$ or selectivity index <3) (11) in any of the remaining 20 subjects. In seven out of the 13 patients with unilateral or bilateral lesions (case numbers 6, 8, 14, 18, 23, 32, and 33), we could not get a more precise diagnosis by AVS (four refused and three had an unsuccessful procedure); nevertheless, we decided to include them in the study.

For statistical evaluation, we considered as treated with mineralocorticoid receptor antagonist those patients (22 out of 34) who took these drugs for at least half of the follow-up time. In six patients, a mineralocorticoid receptor antagonist was the only anti-hypertensive drug taken.

\section{Reevaluation}

The Local Ethics Committee approved the study. All subjects gave their consent to participate in the study and to use clinical data for publication. All patients were studied after withdrawal of interfering therapy for at least 30 days, suspension of the low-salt diet for a week, and after correction of plasma potassium levels if needed. In all patients, basal PAC, PRA, ARR, and PAC after $\mathrm{NaCl}$ test and potassium and creatinine levels were measured. The same criteria as at diagnosis were used to define the presence (persistence) of PA. Furthermore, patients underwent imaging of adrenals by nuclear magnetic resonance (NMR).

\section{Analytical methods}

All the assays were performed in the Central Laboratory of our hospital. PRA and PAC were measured by commercial kits. From 1997 to 2008 , PAC was assessed by RIA (ALDOSTERONE MAIA, Adaltis, Bologna, Italy). The sensitivity of the assay was $6.0 \mathrm{pg} / \mathrm{ml}$. The intraand interassay coefficients of variations $(\mathrm{CV})$ were from 5.76 to $8.16 \%$ and from 11.96 to $14.06 \%$ respectively. PRA was assessed by RIA (ANGIOTENSINA I CT, Radim, Pomezia, Italy). The sensitivity of the assay was $0.15 \mathrm{ng} / \mathrm{ml}$. The intra- and interassay CV were from 4.32 to $7.18 \%$ and from 4.87 to $9.94 \%$ respectively.

From 2008, PAC was assessed by RIA (ALDOCTK-2, DiaSorin, Saluggia, Italy). The sensitivity of the assay 
was $10 \mathrm{pg} / \mathrm{ml}$. The intra- and interassay CV were from 1.7 to $5.3 \%$ and from 3.4 to $7.0 \%$ respectively. PRA was assessed by RIA (RENCTK, DiaSorin, Saluggia, Italy). The sensitivity of the assay was $0.2 \mathrm{ng} / \mathrm{ml}$. The intra- and interassay CV were from 5.4 to $9.9 \%$ and from 7.7 to $11.5 \%$ respectively. All other biochemical variables were assayed in plasma or serum using standard methods.

\section{Statistical analysis}

Statistical analysis was carried out using the application software STATA 10, StataCorp LP (College Station, TX, USA). Mean and percent values of variables at diagnosis and revaluation (patient characteristics, clinical data, and hormonal levels) were compared in the groups with remission or persistence of PA by the Student's $t$-test or Fisher's exact test. Non-normally distributed variables were log-transformed before analysis. Univariate logistic regression was also performed considering confirmation of PA as the binary independent variable.

\section{Results}

When retested, patients were $41-78$ years old $(58 \pm 8.5$; mean \pm s.D.); their demographic and clinical features are shown in Table 1. At revaluation, basal ARR and PAC levels after $\mathrm{NaCl}$ aldosterone were clearly reduced when compared with the same data recorded at diagnosis $(612.4 \pm 724.3$ vs $2251.6 \pm 1496.4, P<0.001$ and $117.0 \pm 122.2$ vs $256.3 \pm 136.0, P<0.001)$.

At reevaluation, only 19 out of 34 patients (56\%) had both basal PAC $>150 \mathrm{pg} / \mathrm{ml}$ and ARR $>400$. Eleven of these patients had a negative confirmatory test (PAC $<100 \mathrm{pg} / \mathrm{ml}$ after $\mathrm{NaCl}$; Fig. 1); therefore, according to our criteria, PA was confirmed only in eight patients (confirmed PA (C-PA), 24\%, case numbers 6, 7, 13, 16, $18,23,28$, and 34), while in 26 PA was not confirmed (NC-PA, 76\%). If the least restrictive criteria suggested by the ES Guideline (11) (i.e. ARR > 200 and PAC after $\mathrm{NaCl}$ above $50 \mathrm{pg} / \mathrm{ml}$ ) were applied, 20 subjects (59\%) were still not affected by PA.

The proportion of NC-PA was even higher if we excluded from the study those patients (case numbers 6 , $8,14,18,23,32$, and 33) in whom an aldosteronesecreting tumor was not excluded with certainty (i.e. patients with an adrenal mass and a not-performed or not-successful AVS). Of the remaining 27 patients, $22(81 \%)$ did not meet our criteria for the diagnosis of $\mathrm{PA}$ at reevaluation.

\section{Predictors of remission of PA}

Variables measured at diagnosis Mean serum potassium levels were significantly higher in NC-PA (3.9 \pm 0.4 vs $3.4 \pm 0.6 \mathrm{mmol} / \mathrm{l}, P=0.03$; Table 2 ); a low potassium concentration (i.e. $<3.5 \mathrm{mmol} / \mathrm{l}$ ) was more frequent in patients with C-PA (4/8 vs $4 / 24$ ), but this result was at the limit of statistical significance $(P=0.07)$. Age at diagnosis $(49.3 \pm 7.9$ vs $48.2 \pm 6.7$ years), presence of unilateral adrenal mass $(20 / 26$ $(77 \%)$ vs $4 / 8(50 \%))$, and reason for referral for PA screening (hypokalemia 4/26 (15\%) vs 3/8 (38\%) and resistant hypertension 10/26 (38\%) vs 4/8 (50\%)) were not significantly associated with NC-PA. Either PAC $(392.2 \pm 193.5$ vs $447.7 \pm 143.6 \mathrm{pg} / \mathrm{ml})$ or ARR $(2333.3 \pm 1519.4$ vs $1986.1 \pm 1485.0)$ at baseline or PAC after confirmatory test $(189.6 \pm 105.7$ vs 255.9 $\pm 163.1 \mathrm{pg} / \mathrm{ml}$ ) was not significantly different in NC-PA. Basal and dynamic PAC of individual cases at diagnosis and retesting are represented in Fig. 1.

Variables measured at reevaluation NC-PA patients were older (age over 60, 17/26 (65\%) vs 1/8 (13\%); $P=0.01)$, showed a longer duration of hypertensive disease $(18 \pm 6.1$ vs $12 \pm 5.2$ years, $P=0.02)$, a longer follow-up $(9.3 \pm 3.3$ vs $5.3 \pm 2.2$ years, $P=0.004)$ and were treated with fewer antihypertensive drugs $(2.1 \pm 1.1$ vs $3.2 \pm 1.9, P=0.05)$. At variance, $\mathrm{NC}-\mathrm{PA}$ patients did not differ from C-PA in BMI (29.6 \pm 4.4 vs $28.4 \pm 5.7)$ and treatment with mineralocorticoid receptor antagonist $(16 / 26(62 \%)$ vs $6 / 8$ (75\%), $P=0.40)$. At reevaluation, four patients were shown to be hypokalemic, and only one had NC-PA.

Other variables Female patients had NC-PA more frequently than males $(15 / 26(58 \%)$ vs $1 / 8(13 \%)$, $P=0.03)$. Family history of hypertension $(24 / 26(92 \%)$ vs $7 / 8(88 \%))$ or early cardiovascular diseases $(2 / 26$ $(8 \%)$ vs $2 / 8(25 \%))$ and the new analytical method of aldosterone assay used after 2008 (7/26 (27\%) vs $1 / 8(13 \%))$ were not associated with remission.

\section{Adrenal morphology}

Adrenal imaging revealed no changes in 27 out of 34 patients; in none of the subjects, the confirmed adrenal lesions showed significant increase during follow-up. Two NC-PA subjects and one C-PA did not show a previously observed adrenal lesion, while four new adrenal lesions appeared. Three of these new lesions were discovered in NC-PA patients and were considered as non-functioning cortical adenomas as screening of cortical hormones and metanephrine secretion was negative. A new lesion was discovered in a C-PA patient who already displayed a mass in the contralateral adrenal at diagnosis. This new picture is currently under investigation.

\section{Discussion}

The main finding of our study is that, some years after diagnosis, most of the patients with aldosteronism after medical treatment have normal basal and dynamic 


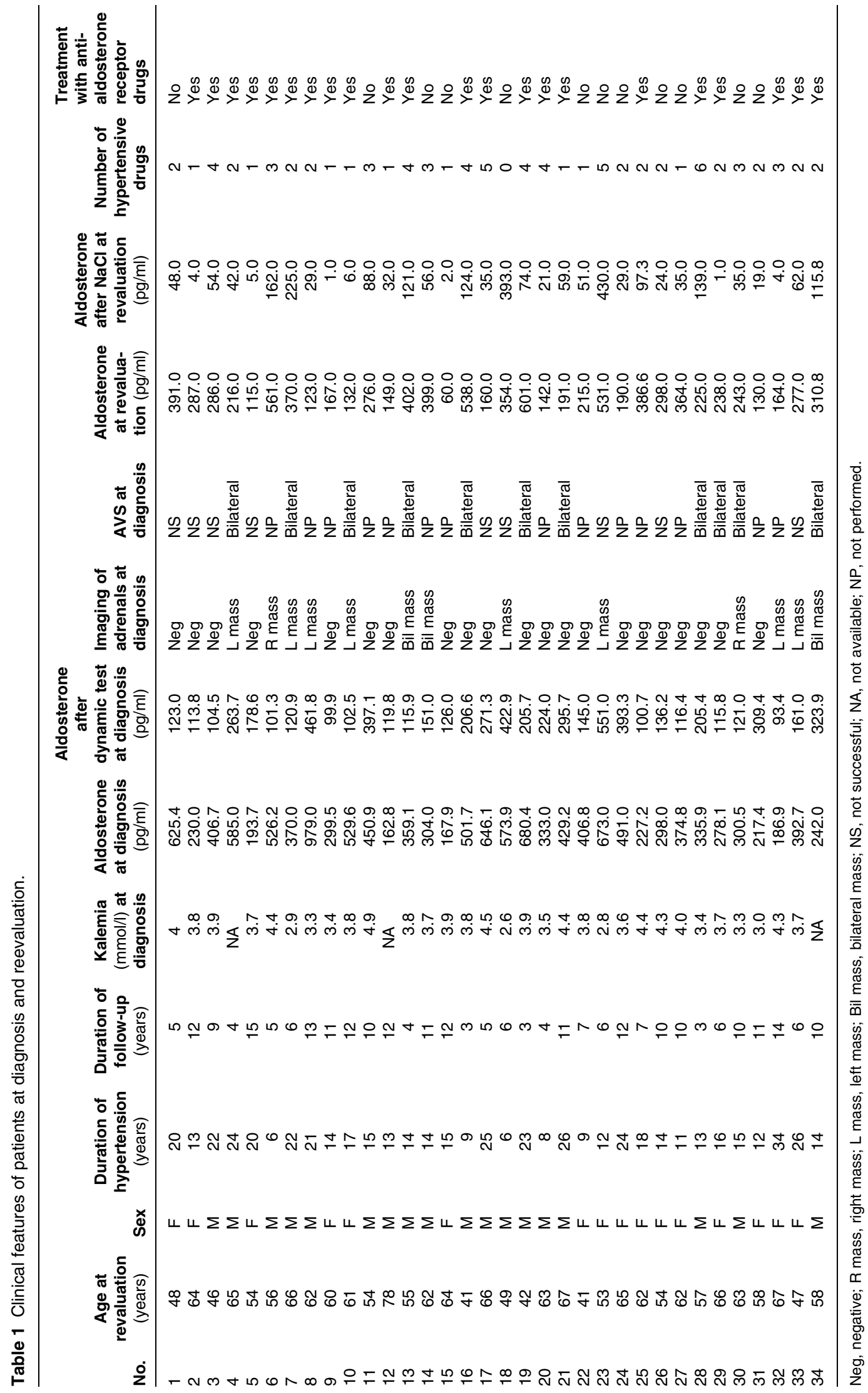




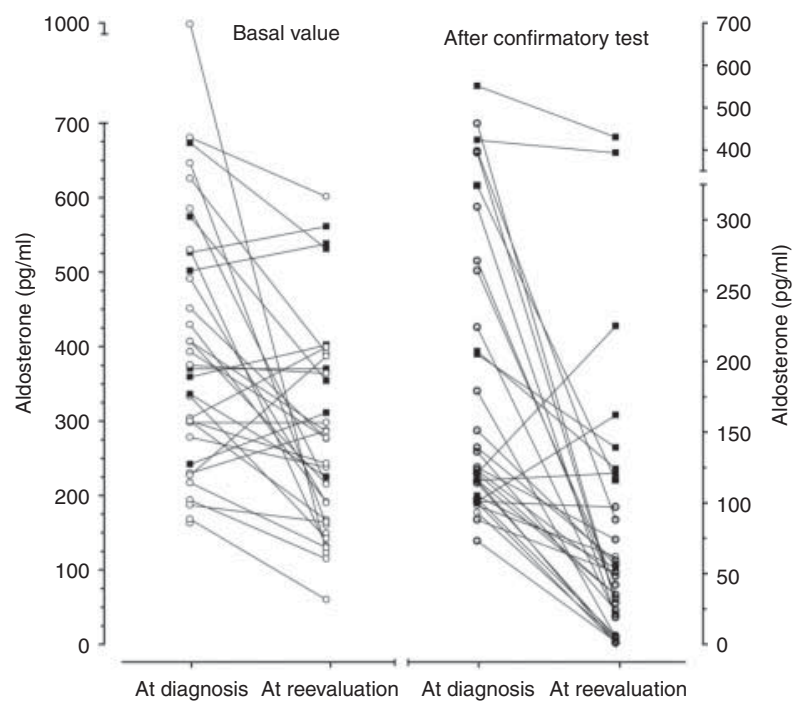

Figure 1 Basal aldosterone levels (left) and aldosterone levels after confirmatory test (right) at diagnosis and at reevaluation. Open circles represent patients with remission and black squares represent patients with persistence of primary aldosteronism at reevaluation.

mineralocorticoid function. More than $75 \%$ of patients previously classified as PA (using restrictive criteria) no longer meet those criteria used for the diagnosis and, even if we apply the least restrictive criteria at reevaluation, more than half of the patients still result 'normalized'. Higher potassium levels at diagnosis, female gender, a longer duration of hypertension, and a longer follow-up seem to predict 'normal' mineralocorticoid secretion.

On the contrary, the study based on the German Conn's Registry (10) reported a normalization of mineralocorticoid secretion in only two out of 37 cases of idiopathic hyperaldosteronism (IHA) at follow-up. This difference is not easy to explain but some differences in protocol of diagnosis or characteristics of the population between the studies could account for the discrepancy. In fact, in the German registry series, i) diagnosis of aldosteronism is based on very low cut-offs both of ARR and aldosterone after saline infusion test, ii) plasma renin concentration instead of PRA is used, iii) in the criteria for 'normalization' blood pressure is also considered, and iv) it is not known whether diagnosis of PA was made by screening or case finding in high-prevalence groups.

We are aware that medically treated aldosteronism cannot be considered a homogeneous group of patients. Most of the patients we studied can be defined as idiopathic hyperaldosteronism. In a minor portion, the diagnosis remains undefined for technical problems (unsuccessful AVS) or personal choice of the patients (refusal of AVS). It is a serious challenge to recognize an aldosterone-secreting adenoma with good confidence because of the small size of the adenoma, the frequency of non-secreting adrenal adenoma, and the high rate of inconclusive adrenal venous sampling. In any case,

Table 2 Variables in patients with non-confirmed primary aldosteronism (NC-PA) or confirmed PA (C-PA).

\begin{tabular}{|c|c|c|c|c|c|}
\hline & NC-PA $(n=26)$ & C-PA $(n=8)$ & $P^{a}$ & OR $(\mathrm{Cl})$ & $P^{\mathrm{b}}$ \\
\hline \multicolumn{6}{|l|}{ Variables registered at diagnosis } \\
\hline Kalemia $(\mathrm{mmol} / \mathrm{l})$ & $3.9 \pm 0.4$ & $3.4 \pm 0.6$ & 0.03 & $7.77(1.02-54.6)$ & 0.05 \\
\hline Hypokalemia & $4(17 \%)$ & $4(50 \%)$ & 0.07 & $0.18(0.03-1.04)$ & 0.06 \\
\hline Basal PAC (pg/ml) & $392.2 \pm 193.5$ & $447.7 \pm 143.6$ & 0.46 & $0.99(0.99-1.00)$ & 0.45 \\
\hline ARR & $2333.3 \pm 1519.4$ & $1986.1 \pm 1485$ & 0.57 & $1.00(0.99-1.00)$ & 0.58 \\
\hline PAC after test $(\mathrm{pg} / \mathrm{ml})$ & $189.6 \pm 105.7$ & $255.9 \pm 163.1$ & 0.18 & $0.99(0.99-1.00)$ & 0.19 \\
\hline Unilateral adrenal mass & $6(23 \%)$ & $4(50 \%)$ & 0.15 & $0.3(0.06-1.58)$ & 0.16 \\
\hline \multicolumn{6}{|l|}{ Reason for referral } \\
\hline Hypokalemia & $4(15 \%)$ & $3(38 \%)$ & 0.17 & $0.11(0.01-1.40)$ & 0.09 \\
\hline Resistant hypertension & $10(38 \%)$ & $4(50 \%)$ & & $0.21(0.02-1.18)^{\mathrm{c}}$ & 0.19 \\
\hline Age at diagnosis (years) & $49.3 \pm 7.9$ & $48.2 \pm 6.7$ & 0.72 & $1.01(0.91-1.13)$ & 0.71 \\
\hline \multicolumn{6}{|l|}{ Variables registered at reevaluation } \\
\hline Over 60 years at reevaluation & $17(65 \%)$ & $1(13 \%)$ & 0.01 & $13.2(1.40-124)$ & 0.02 \\
\hline Years of hypertension & $18 \pm 6.1$ & $12 \pm 5.2$ & 0.02 & $1.24(1.02-1.52)$ & 0.03 \\
\hline Years of follow-up & $9.3 \pm 3.3$ & $5.3 \pm 2.2$ & $<0.01$ & $1.52(1.08-2.15)$ & 0.02 \\
\hline Follow-up over 7.5 years & $17(65 \%)$ & $1(13 \%)$ & 0.01 & $13.2(1.40-124)$ & 0.02 \\
\hline Same PAC assay as at diagnosis & $7(27 \%)$ & $1(13 \%)$ & 0.38 & $2.58(0.27-24.9)$ & 0.41 \\
\hline No. of antihypertensive drugs & $2.1 \pm 1.1$ & $3.2 \pm 1.9$ & 0.05 & $0.57(0.32-1.04)$ & 0.07 \\
\hline Aldosterone receptor antagonists & $16(62 \%)$ & $6(75 \%)$ & 0.40 & $0.53(0.09-3.18)$ & 0.49 \\
\hline BMI & $29.6 \pm 4.4$ & $28.4 \pm 5.7$ & 0.56 & $1.06(0.88-1.26)$ & 0.55 \\
\hline \multicolumn{6}{|l|}{ Other variables } \\
\hline Male & $11(42 \%)$ & $7(87 \%)$ & 0.03 & $0.10(0.01-0.98)$ & 0.05 \\
\hline Family history of hypertension & $24(92 \%)$ & $2(88 \%)$ & 0.57 & $1.71(0.13-21.9)$ & 0.68 \\
\hline Family history of early CVD & $2(8 \%)$ & $2(25 \%)$ & 0.23 & $0.25(0.29-2.16)$ & 0.21 \\
\hline
\end{tabular}

aStudent's t-test or Fisher's exact test.

bUnivariate logistic regression.

${ }^{\mathrm{C} V s}$ other reasons.

CVD, cardio-vascular disease. 
despite these limitations, we decided to maintain in the study all those patients who did not undergo surgery. Yet, we studied only patients diagnosed with 'strict' biochemical criteria: we made diagnosis of PA only when all the following conditions were satisfied: ARR over 400 and plasma aldosterone levels over $150 \mathrm{pg} / \mathrm{ml}$ and over $100 \mathrm{pg} / \mathrm{ml}$ after $\mathrm{NaCl}$ test. These precautions should protect from false-positive results of increased and not suppressible aldosterone secretion. Nevertheless, $50-75 \%$ of these patients did not confirm primary hypersecretion when studied at least 3 years after diagnosis. A simple 'regression toward the mean' phenomenon seems unlikely; when patients were retested, aldosterone levels were clearly reduced both in basal condition and after the suppression test. Alternatively, we should assume that our patients were not correctly diagnosed at the beginning, implying that the official criteria of diagnosis provide a high number of false-positive results.

Patients were evaluated at diagnosis and retested in the same conditions. In order to avoid wrong estimation of the biochemical tests, patients were always studied during normal sodium intake, normal potassium levels, and after a wash-out of the same interfering drugs (diuretics, mineralocorticoids receptor antagonists, $\beta$-blockers, ACE inhibitors, angiotensin II receptor antagonists, and clonidine). Long-acting dihydropyridinic and non-dihydropyridinic calcium-antagonists were permitted when pressure control was poor with $\alpha$-blockers. It is a shared opinion that dihydropyridinic calcium channel blockers do not significantly affect aldosterone secretion, causing mainly an increase in PRA, which rarely gives false negatives $(5,11,12)$. Furthermore, patients treated with these drugs at reappraisal were almost the same as at diagnosis (13 and 15 respectively), so it is very unlikely that the use of these drugs at reevaluation accounted for the low rate of C-PA.

The only difference from diagnosis and retesting was that, at diagnosis, 16 patients underwent fludrocortisone instead of $\mathrm{NaCl}$ as suppression test, as suggested by Gordon et al. (12). At reevaluation, for obvious reasons of costs and convenience, we had always chosen to run the $\mathrm{NaCl}$ test, now considered by most authors a reliable alternative to the more expensive and complex fludrocortisone test (11). Moreover, according to our experience, the $\mathrm{NaCl}$ test has proved to perform no differently to the fludrocortisone test (14).

Years after diagnosis of PA, a high rate of 'normalization' of aldosterone secretion was reported in 2005 by Armanini et al. (8) but, differently from the present study, they only recorded basal mineralocorticoid function in patients with idiopathic aldosteronism. In that study, 15 patients with long-term (3-24 years) PA were studied 1 month after withdrawal of therapy with potassium canrenoate. ARR was increased only in three subjects and there was a significant inverse correlation between ARR and the number of years of therapy with potassium canrenoate (8). In a later work, the same authors extended the hormonal control to three more patients, treated for more than 10 years with potassium canrenoate and then suspended for at least 5 years. In those three patients, ARR was normal (9). Our reappraisal adds the evidence that patients with normal basal mineralocorticoid secretion also have a normal suppressibility of aldosterone production, thus indicating a complete normalization of the previously diagnosed PA. Moreover, recently, Yoneda et al. (13) reported a case of remission of unilateral primary hyperaldosteronism during treatment with antagonists of mineralocorticoid receptor, indicating that spironolactone is not only able to antagonize the receptor but also to decrease aldosterone synthetic activity, and speculating that it may produce remission of hyperaldosteronism. The above-mentioned studies suggested that long-term therapy with mineralocorticoid receptors blockers could have played a role in the remission of PA $(8,9,13)$. It is known that spironolactone can directly inhibit the synthesis of aldosterone in the zona glomerulosa of the adrenals (15) and this observation is indirectly proven by the histological finding of spironolactone bodies in the glomerulosa of patients with PA treated with the drug (16). Furthermore, prolonged treatment with aldosterone receptor blockers can affect the sensitivity of adrenal glands to angiotensin II or the tropic factors at the level of the glomerulosa, which can be involved in the pathogenesis of IHA (8). Yet, long-term therapy with mineralocorticoid receptors blockers is able to increase renin activity due to volume depletion and this effect can restore a normal responsiveness of glomerulosa to angiotensin II. However, our data challenge the interpretation that the long-term remission of aldosterone hypersecretion is due to aldosterone antagonists. In fact, long-term therapy with these drugs proved to be a poor predictor of remission of aldosterone hypersecretion and furthermore renin activity was low or normal in most cases.

In our study, aldosterone levels at diagnosis do not seem important in targeting patients with future remission of PA. In fact, plasma aldosterone levels and ARR at diagnosis were not significantly lower in patients with remission of PA. Similarly, the evidence of a unilateral adrenal mass did not predict the persistence of hyperaldosteronism. This is not surprising as false-positive images of secreting adenomas are frequently reported (17).

Interestingly, in our study, age at reevaluation and duration of follow-up seem to be significant predictors of remission of PA. It is known that PRA and aldosterone progressively decrease with age $(18,19)$ so that we can speculate that even in subjects with apparent mineralocorticoid excess, the aging process might impair aldosterone secretion. Aging is associated with reduced adrenal responsiveness to angiotensin II, contributing to lower production of aldosterone and imbalance of sodium homeostasis (19). These factors could be involved in the resolution of mild forms of PA, 
considering that hypersensitivity of glomerulosa to angiotensin is suggested to play a role in the pathogenesis of the disease (20).

In addition, we should not forget that biochemical assays at retesting were performed several years after the first investigation so that most of our patients $(26$ out of 34 cases) have been retested using a different assay from that used at the time of diagnosis. We are aware that both current and previous aldosterone and renin activity assays are not perfectly reliable (21). Schirpenbach et al. (22) clearly demonstrated markedly different results in a comparison of four different aldosterone assays. It is possible in some cases that a poor comparability of laboratory methods $(22,23)$ for the determination of aldosterone and renin activity accounted for non-confirmation of mineralocorticoid hypersecretion. However, it has to be underlined that only one out of the eight cases retested with the same method as at diagnosis had PA confirmed, suggesting that changing of the analytical method was probably not a major problem.

In conclusion, our results suggest that in the minority of PA after medical treatment, the biochemical picture persists over years, whereas in the majority primary mineralocorticoid hypersecretion declines with age. Understanding whether the 'remission' of PA is a true event, due either to specific drug treatment or determined by a biological spontaneous phenomenon, deserves further prospective studies. The ideal study to confirm PA remission should be performed in a larger population of patients and include a prospective evaluation of the responses to standardized biochemical tests of hypersecretion and suppressibility of aldosterone secretion, either in patients without adrenal lesion or with adrenal mass judged non-secreting adenoma. The clinical picture should also be revised periodically in parallel with biochemical status, particularly for what concerns blood pressure control, target organ damage and metabolic parameters known or supposed to be associated with mineralocorticoid hyperfunction (sodium, potassium, creatinine, glucose levels, and body composition); major cardiovascular events should also be recorded during the study.

If confirmed, the observation of a true and frequent decline with time of idiopathic PA could have important clinical implications for a clinical condition now considered quite common: for instance, it could justify periodical diagnostic reassessments at least when the clinical picture is not so severe.

\section{Declaration of interest}

The authors declare that there is no conflict of interest that could be perceived as prejudicing the impartiality of the research reported.

\section{Funding}

This research did not receive any specific grant from any funding agency in the public, commercial or not-for-profit sector.

\section{References}

1 Viera AJ \& Neutze DM. Diagnosis of secondary hypertension: an age-based approach. American Family Physician $2010 \mathbf{8 2}$ 1471-1478.

2 Young WF Jr. Minireview: primary aldosteronism-changing concepts in diagnosis and treatment. Endocrinology $2003 \mathbf{1 4 4}$ 2208-2213. (doi:10.1210/en.2003-0279)

3 Rossi GP, Bernini G, Caliumi C, Desideri G, Fabris B, Ferri C, Ganzaroli C, Giacchetti G, Letizia C, Maccario M et al. A prospective study of the prevalence of primary aldosteronism in 1,125 hypertensive patients. Journal of the American College of Cardiology 200648 2293-2300. (doi:10.1016/j.jacc.2006.07.059)

4 Biglieri EG. Primary aldosteronism due to primary adrenal hyperplasia. In The Adrenal Gland and Hypertension, pp 353-356. Eds F Mantero, EG Biglieri, JW Funder \& B Scoggins, New York: Raven Press, 1985.

5 Kaplan NM. The current epidemic of primary aldosteronism: causes and consequences. Journal of Hypertension 200422 863-869. (doi:10.1097/00004872-200405000-00001)

6 Irony I, Biglieri EG \& Kater CE. The adrenocortical hormones in hypertensive disorders. In Endocrine Mechanism in Hypertension, pp 1-18. Eds JH Laragh, BM Brenner \& NM Katler, New York: Raven Press, 1989.

7 Pozzan GB, Armanini D, Cecchetto G, Opocher G, Rigon F, Fassina A \& Zacchello F. Hypertensive cardiomegaly caused by an aldosterone-secreting adenoma in a newborn. Journal of Endocrinological Investigation 19972 86-89.

8 Armanini D, Scaroni C, Mattarello MJ, Fiore C, Albiger N \& Sartorato P. Idiopathic primary hyperaldosteronism: normalization of plasma aldosterone after one month withdrawal of long-term therapy with aldosterone-receptor antagonist potassium canrenoate. Journal of Endocrinological Investigation 200528 236-240.

9 Armanini D, Fiore C \& Pellati D. Spontaneous resolution of idiopathic aldosteronism after long-term treatment with potassium canrenoate. Hypertension $2007 \quad \mathbf{5 0}$ e69-e70. (doi:10.1161/HYPERTENSIONAHA.107.096925)

10 Fischer E, Beuschlein F, Degenhart C, Jung P, Bidlingmaier M \& Reincke M. Spontaneous remission of idiopathic aldosteronism after long-term treatment with spironolactone: results from the German Conn's Registry. Clinical Endocrinology $20127 \mathbf{7 6}$ 473-477. (doi:10.1111/j.1365-2265.2011.04243.x)

11 Funder JW, Carey RM, Fardella C, Gomez-Sanchez CE, Mantero F, Stowasser M, Young WF Jr, Montori VM \& Endocrine Society . Case detection, diagnosis, and treatment of patients with primary aldosteronism: an endocrine society clinical practice guideline. Journal of Clinical Endocrinology and Metabolism 2008933266 3281. (doi:10.1210/jc.2008-0104)

12 Gordon RD. Primary aldosteronism. Journal of Endocrinological Investigation $1995 \mathbf{1 8}$ 495-511.

13 Yoneda T, Demura M, Takata H, Kometani M, Karashima S, Yamagishi M \& Takeda Y. Unilateral primary aldosteronism with spontaneous remission after long-term spironolactone therapy. Journal of Clinical Endocrinology and Metabolism 201297 1109-1113. (doi:10.1210/jc.2011-2563)

14 Mulatero P, Milan A, Fallo F, Regolisti G, Pizzolo F, Fardella C, Mosso L, Marafetti L, Veglio F \& Maccario M. Comparison of confirmatory tests for the diagnosis of primary aldosteronism. Journal of Clinical Endocrinology and Metabolism 200691 2618-2623. (doi:10.1210/jc.2006-0078)

15 Mantero F, Armanini D \& Urbani S. Antihypertensive effect of spironolactone in essential, renal and mineralocorticoid hypertension. Clinical Science and Molecular Medicine. Supplement 197345 219S-224S.

16 Cohn D, Jackson RV \& Gordon RD. Factors affecting the frequency of occurrence of spironolactone bodies in aldosteronomas and non-tumorous cortex. Pathology 198315 273-277. (doi:10.3109/00313028309083505) 
17 Young WF, Stanson AW, Thompson GB, Grant CS, Farley DR \& van Heerden JA. Role for adrenal venous sampling in primary aldosteronism. Surgery 2004136 1227-1235. (doi:10.1016/ j.surg.2004.06.051)

18 Tsunoda K, Abe K, Goto T, Yasujima M, Sato M, Omata K, Seino M \& Yoshinaga K. Effect of age on the renin-angiotensin-aldosterone system in normal subjects: simultaneous measurement of active and inactive renin, renin substrate, and aldosterone in plasma. Journal of Clinical Endocrinology and Metabolism $1986 \mathbf{6 2}$ 384-389. (doi:10.1210/jcem-62-2-384)

19 Belmin J, Lévy BI \& Michel JB. Changes in the renin-angiotensinaldosterone axis in later life. Drugs \& Aging 19945 391-400. (doi:10.2165/00002512-199405050-00007)

20 Lim PO, Young WF \& MacDonald TM. A review of the medical treatment of primary aldosteronism. Journal of Hypertension 200119 353-361. (doi:10.1097/00004872-200 103000-00001)
21 Stowasser M. Update in primary aldosteronism. Journal of Clinical Endocrinology and Metabolism 200994 3623-3630. (doi:10.1210/jc.2009-1399)

22 Schirpenbach C, Seiler L, Maser-Gluth C, Beuschlein F, Reincke M \& Bidlingmaier M. Automated chemiluminescence-immunoassay for aldosterone during dynamic testing: comparison to radioimmunoassay with and without extraction steps. Clinical Chemistry 2006 52 1749-1755. (doi:10.1373/clinchem.2006.068502)

23 Tomaschitz A \& Pilz S. Aldosterone to renin ratio - a reliable screening tool for primary aldosteronism? Hormone and Metabolic Research 201042 382-391. (doi:10.1055/s-0030-1248326)

Received 17 October 2012

Revised version received 11 January 2013

Accepted 15 January 2013 